\title{
Prospective regenerative medicine therapies for obstetric trauma-induced fecal incontinence
}

\begin{abstract}
Fecal incontinence is a major public health issue that has yet to be adequately addressed. Obstetric trauma and injury to the anal sphincter muscles are the most common cause of fecal incontinence. New therapies are emerging aimed at repair or regeneration of sphincter muscle and restoration of continence. While regenerative medicine offers an attractive option for fecal incontinence there are currently no validated techniques using this approach. Although many challenges are yet to be resolved, the advent of regenerative medicine is likely to offer disruptive technologies to treat and possibly prevent the onset of this devastating condition. This article provides a review on regenerative medicine approaches for treating fecal incontinence and a critique of the current landscape in this area.
\end{abstract}

Keywords: biomaterials $\bullet$ cell therapy $\bullet$ fecal incontinence $\bullet$ muscle $\bullet$ obstetric trauma

- regenerative medicine $\bullet$ tissue engineering

\section{Pelvic floor maintenance of continence}

Fecal incontinence (FI) is defined as involuntary loss of fecal material [1]. The anal sphincter consists of two muscular components. The internal anal sphincter (IAS), which composed of a torus of smooth muscle, measures $0.3-0.5 \mathrm{~cm}$ in thickness and extends approximately $2.5 \mathrm{~cm}$ along the anal canal. The IAS is surrounded by the external anal sphincter (EAS), which is composed of skeletal muscle, and measures $0.6-1.0 \mathrm{~cm}$ in thickness and $8-10 \mathrm{~cm}$ in length (Figure 1) [2-4]. The two muscles contribute to approximately 70 and $30 \%$ of the basal resting tone, respectively, working in co-ordination to provide voluntary and involuntary control of continence and facilitate controlled closure of the anal canal $[4,5]$. The EAS is slow-twitch, type I striated muscle under voluntary control of sensory pudendal nerves in the anal canal and is not innervated by the enteric nervous system. Few other skeletal muscle structures in the human body share the circular layout without tendons or direct connection to bone, while also generating tone at rest. The IAS is a ring of involuntary smooth muscle, controlled by the autonomic nervous system providing tonic contraction [6]. Through tonic contraction, the IAS is principally responsible for anal continence at rest, whereas the EAS increases anal pressure in response to increased intra-rectal or intraabdominal pressure. Impairment of anal sphincter function leads to reduced resting pressure and is a cause of FI [7]. Additionally, the levator ani muscle which constitutes the pelvic floor, and puboretalis muscle, function as a sling to support the anorectal angle and contribute to continence [8]. Anal canal cushions are highly vascularized and interdigitated structures that provide a thickened lining and have been postulated to contribute to the resting pressure [9]. However, this view is not accepted universally and studies have found no difference to continence despite removal of anal cushions posthemorrhoidectomy $[10,11]$.

Defecation is controlled by coordinated sensory-motor innervation that uses nerves and muscles of the pelvic floor, EAS and
Nina Parmar ${ }^{1}$, Lalit Kumar' ${ }^{2}$, Anton Emmanuel ${ }^{2}$ \& Richard M Day*,1

'Applied Biomedical Engineering Group, University College London, 21 University Street, London, WC1E 6JJ, UK ${ }^{2} \mathrm{GI}$ Physiology Unit, University College Hospital, London, UK

*Author for correspondence: r.m.day@ucl.ac.uk 


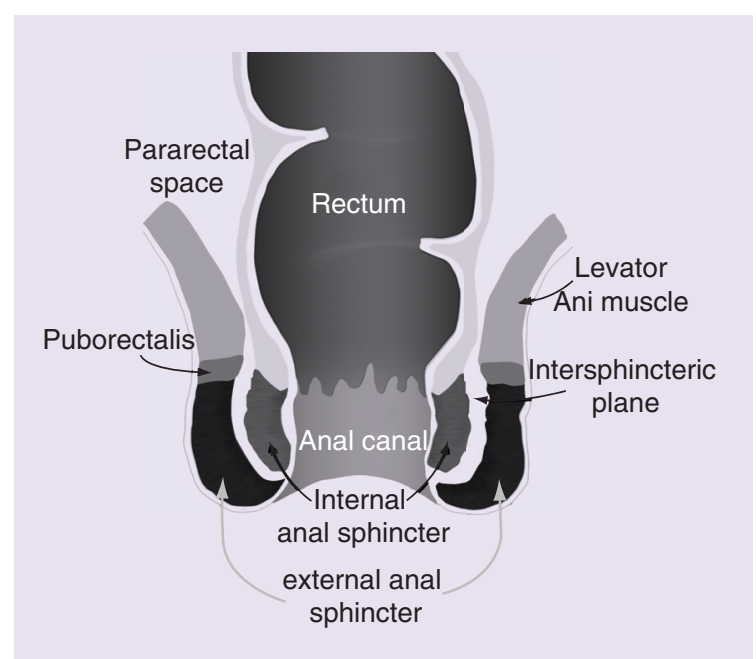

Figure 1. Anorectal anatomy.

IAS. When the rectal threshold volume is reached, stretch receptors are activated in the myenteric plexus in the rectal walls along the pelvic floor, initiating a sensation of urge. Defecation reflexes, in the form of peristaltic waves in the descending colon and rectum, force feces to the anus. Peristaltic waves towards the anus result in inhibitory signals from the myenteric plexus to cause the IAS to relax. If the EAS is also voluntarily relaxed at the same time, defecation occurs [12]. FI occurs when one or more of these mechanisms are disrupted and other mechanisms fail to compensate.

\section{Current treatment}

Treatment for FI varies with etiology. In the case of an obstetric injury, management of FI ranges from holistic approaches based on nutrition and psychological health, to surgery (Table 1). However, delayed and under-reporting of symptoms by patients in addition to a huge disparity in long-term monitoring of obstetric tears across the maternity care units makes this a challenging problem to tackle. Moreover, a UK-based study reported that almost two-thirds of clinicians practicing obstetrics felt inadequately trained in the training and management of obstetric trauma-induced anal sphincter injuries [13].

\section{Obstetric trauma-induced FI}

Obstetric trauma is a well-recognized cause of FI in women [14]. The prevalence of post partum FI reported in literature has a wide range varying from 3 to $29 \%$ [14-16]. Obstetric trauma-induced FI is attributed to two main causes: injury to pudendal nerve, which supplies the EAS and pelvic floor muscles [17], or to the anal sphincter muscles (EAS, IAS or both). Anal sphincters, being more amenable to treatment compared with the pudendal nerve, have been the main focus of management therapies directed towards controlling post partum FI. Although damage to the anal sphincter complex is common during vaginal delivery, it remains clinically undetected in $12-35 \%$ of patients [18]. Studies have revealed that a significant proportion of women with third- or fourth-degree tears become incontinent. A systematic review by Dudding et al. stated that up to two-thirds of women who sustain a recognized third-degree tear during delivery subsequently suffer with FI decades after childbirth [18,19]. There is a clear and unmet need for new approaches in the management and treatment of FI, which has been limited due to under-reporting and inaccurate demographics of FI.

This review focuses on regenerative medicine approaches for restoring the physiological function of sphincter muscle that is of potential clinical value. The review also aims to provide a critique of the current landscape for regenerative medicine being applied to obstetric trauma-induced FI.

\section{Regenerative medicine}

Regenerative medicine aims to restore functional tissue that is impaired due to aging, disease or injury. This promising field holds the potential for regeneration of impaired tissues by stimulating healing of the damaged tissue or by allowing growth of tissues and organs in the laboratory, which can then be implanted into the body. The common approaches in this field include using cell therapy, biomaterials, a combination of both, or tissue engineering (TE).

Cell therapy involves the introduction of cells into a tissue with the aim of restoring lost tissue function.

Table 1. Current treatment options available for fecal incontinence.

\begin{tabular}{|lll|}
\hline Noninvasive & Minimally invasive & Invasive \\
\hline Difestyle modification & Biofeedback & Anal sphincter repair \\
\hline Drugs & Bulking agents & Artificial anal sphincters \\
\hline Pelvic floor exercises & Injectable microspheres & Stoma \\
\hline \multicolumn{2}{r}{} & \\
\hline & Cell therapy & \\
\hline
\end{tabular}


Transplanted cells can modulate the host's reparative response or may regenerate into new tissue itself. However, in cases of large tissue loss cell therapy alone is often not sufficient and there is a need for biomaterials to provide a provisional scaffold structure that can protect the cells during delivery and increase the likelihood of engraftment to form functional tissue. Biomaterials do not only facilitate targeted cell delivery and integration with host tissue, but can also guide the healing process. Another promising approach is TE, which involves growth of functional organs in vitro that are subsequently implanted into the body. This technique utilizes three basic tools: cells, scaffolds and growth stimulatory signals (which maybe chemical or physical in nature). TE has received a lot of attention as an ideal treatment for many conditions, but it largely remains at the research stage.

Regenerative medicine offers an ideal solution for sphincter muscle damage caused by obstetric trauma where there is no associated neurological damage. It is feasible that many of these techniques, discussed in the ensuing sections, might be suitable for managing FI secondary to obstetric anal sphincter trauma.

\section{Smart biomaterials}

Basic TE principles rely on the use of biologically compatible material to deliver or initiate regeneration of tissue. Smart biomaterial scaffolds are made from a variety of materials to form $3 \mathrm{D}$ structures that can also be used to provide bioactive cues for cells for processes such as facilitation of adhesion, proliferation and differentiation of cells. Bioactive scaffolds are designed to release chemical signals, such as chemokines and growth factors, into the surrounding environment. The $3 \mathrm{D}$ structures can be designed to have specific degradation rates, pore size, porosity and surface topographies for optimal cell and tissue interaction. Material surface topography has been found to have a direct influence on the attachment and phenotype of cells to biomaterials. For example, studies with osteoblasts have shown they can be directed towards a proliferative phenotype when grown on a smooth surface compared with cells grown on a rough surface [20].

Injectable scaffolds are increasingly popular due to their macroscopic 3D properties for tissue regrowth and a microscopic framework to provide localized cell growth and tissue integration [21]. Appropriate polymer selection and fabrication method of the scaffold is important to mimic endogenous tissue physiology or encourage extracellular matrix deposition for clinical applications in translational research. The different type of polymers along with their advantages and drawbacks are summarized in Table 2.

Natural polymers such as collagen-I and fibrin have been investigated for skeletal muscle regeneration. Collagen-I has been preferentially selected as a suitable biomaterial for applications such as striated muscle regeneration due to it being a predominant constituent of the extracellular matrix in natural skeletal muscle tissue, hence its contribution to myoblast cell proliferation and differentiation. A main advantage is its elasticity during muscle contraction, which closely matches the physiological properties in skeletal muscle [22]. However, rapid shrinkage of pure collagen gels in vitro and poor stability in vivo limits their application [23]. Collagen sponges and hydrogels have been investigated for their ability to regenerate functional tissue [24,25]. A study by Beier et al. in which myoblast cells were attached to electrospun pure collagen-I demonstrated high myoblast proliferation rate in a controlled orientation [26].

Fibrin is another material that has been explored for constructing matrices. Increased amounts of growth factors, such as VEGF, IGF1 and cytokine IL-1 are found in the presence of fibrin [27]. Upregulation of these signaling molecules may encourage host tissue remodeling and repair in the presence of fibrin. How-

Table 2. Examples of different types of polymer biomaterials and their advantages or disadvantages.

\begin{tabular}{|c|c|c|}
\hline Type & Advantage & Disadvantage \\
\hline Natural polymers & Biocompatible & Poor stability in vitro \\
\hline Collagen & Bioresorbable & Rapid shrinkage \\
\hline Fibrin & Low immunogenicity & Migration from implantation site \\
\hline Synthetic polymers & $\begin{array}{l}\text { Improved stability in vivo compared with } \\
\text { natural polymers }\end{array}$ & $\begin{array}{l}\text { Elicit a greater immunogenic } \\
\text { response compared with natural } \\
\text { polymers }\end{array}$ \\
\hline $\begin{array}{l}\text { Poly-(lactic acid); poly- } \\
\text { (glycolic acid); poly-(lactic- } \\
\text { co-glycolic acid) }\end{array}$ & $\begin{array}{l}\text { Poly-(lactic acid), poly-(glycolic acid), } \\
\text { poly-(lactic-co-glycolic acid)-controllable } \\
\text { degradation rates both in vitro and in vivo } \\
\text { compared with natural polymers }\end{array}$ & - \\
\hline
\end{tabular}


ever, fibrin has shown poor stability in vitro beyond 4 weeks, which may not permit comprehensive skeletal muscle regeneration in obstetric trauma patients with a third of fourth degree tear [28-30].

Synthetic biomaterials such as poly-(lactic acid) (PLA), poly-(glycolic acid) (PGA), and their copolymer poly-(lactatic-co-glycolic acid) (PLGA) have improved stability and degradation rates both in vitro and in vivo compared with many natural polymers. Synthetic polymer scaffolds can be made with optimal features such as mechanical integrity to support tissue ingrowth, high porosity to promote nutrient and gas exchange, controlled degradation, and release of bioactive agents over predetermined lengths of time.

A localized increase of growth factors at the site of injury may promote vascularization and regeneration of skeletal muscle tissue. However, bioactive factors to promote angiogenesis and tissue growth alone may be insufficient to promote full tissue restoration of the EAS muscle. The use of smart biomaterials in conjunction with cell delivery or engineered tissue construct implantation could improve neovascularization and enhance engraftment of delivered cells or tissue. Vascularization is thought to be instrumental to the survival of tissue engineered constructs since gaseous exchange and nutrient diffusion in tissues are restricted to distances of no more than $200 \mu \mathrm{m}$. The lack of a vascular network may result in cells at the center of the engineered tissue becoming hypoxic, which may lead to a necrotic core and loss of functional tissue. Encapsulation of anti-scarring agents, for example, isoforms of TGF $\beta$ within biomaterials to be delivered with cells or tissue may promote host tissue engraftment of implanted tissue for improved function [31].

\section{Cell therapy}

Many of the proposed regenerative medicine approaches for FI have been inspired by recent advances made in therapies for urinary incontinence and adapted for application to the anal sphincter. Autologous muscle progenitor cells have been shown to actively regenerate injured striated muscle of the urethral sphincter in rats. Injury was induced using electrocoagulation and urodynamic testing results confirmed that $41 \%$ of sphincter function was restored within 1 month of muscle cell implantation [32]. Histology revealed replacement of mature myofibers, as well as restoration of innervated units in the regenerated sphincter muscle. This study demonstrates the feasibility of minimally invasive delivery of muscle progenitor cells to striated sphincter muscle with discrete injury. The use of electrocoagulation to simulate obstetric injury irreversibly destroyed host myofibers and satellite cell activation. This type of injury is not entirely representative of obstetric injury, with irreversible myofiber destruction possibly limiting the capacity of the delivered cells to engraft and facilitate host tissue. Furthermore, cell delivery at 37 days postinjury was not conducted under guidance, thus introducing the possibility of cell misplacement and variability between recipients. To our knowledge, five US FDA-approved autologous cell therapy clinical trials targeting urinary incontinence have been performed to date. These have shown encouraging results and are summarized in Table 3.

The first reported cell therapy clinical study for FI was performed by Frudinger et al. Autologous myoblasts were injected into the EAS of women with FI caused by obstetric trauma. Symptom duration ranged from 1 to 30 years in these patients prior to myoblast delivery. At 12 months follow-up there was an improvement in Wexner score that had decreased by a mean of 13.7 units and overall quality of life scores had improved by a median of 30 points. Although there was a significant increase in anal squeeze pressure at 1 and 6 month, it was not sustained at 12 months postinjection [34]. This might suggest that the delivered cells provided little or no regeneration of the scarred anal sphincter muscle. The cause of this is unknown, but could be related to inefficient delivery and engraftment of viable cells into the host tissue. In vitro expansion of cells in 2D culture may impact on their behavior when delivered in vivo. The cells must first be detached and placed into a suspension before delivery to the muscle as an injected bolus. Without sufficient vascularization many of the cells are unlikely to survive [41]. Itescu et al. have shown successful engraftment of autologous skeletal myoblasts delivered into injured myocardium, but survival of the implanted cells may be affected by alteration to their immunogenicity due to prolonged ex vivo culture [42]. Hodgetts et al. showed that 90\% of cultured myoblasts die within $1 \mathrm{~h}$ of intravenous transplantation into dystrophic mice. The mechanisms attributed to this were:

- Natural killer cells responding to immunogenic antigens on transplanted myoblasts altered by $2 \mathrm{D}$ cell culture conditions;

- 'Natural' host antibodies, which activate complement via a classical pathway;

- Binding of donor antigen to host antigen-presenting cells to present donor antigens to $\mathrm{T}$ cells $[42,43]$.

For cell therapy to succeed as a regenerative medicine therapy for incontinence, survival of transplanted cells and limitation of immune response may need to be further optimized.

For urinary incontinence, preclinical cell therapy studies have indicated autologous stem cells can restore 


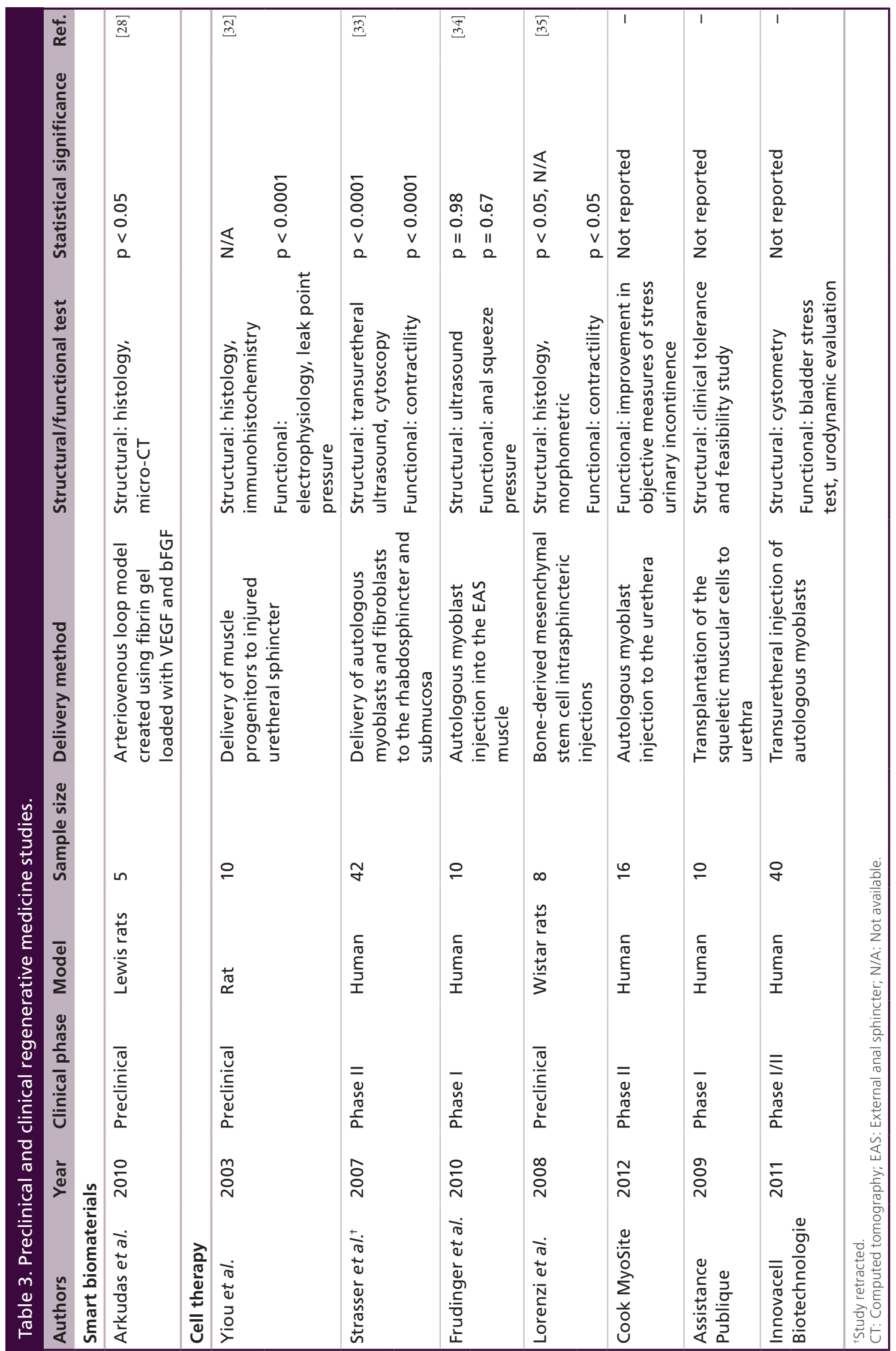




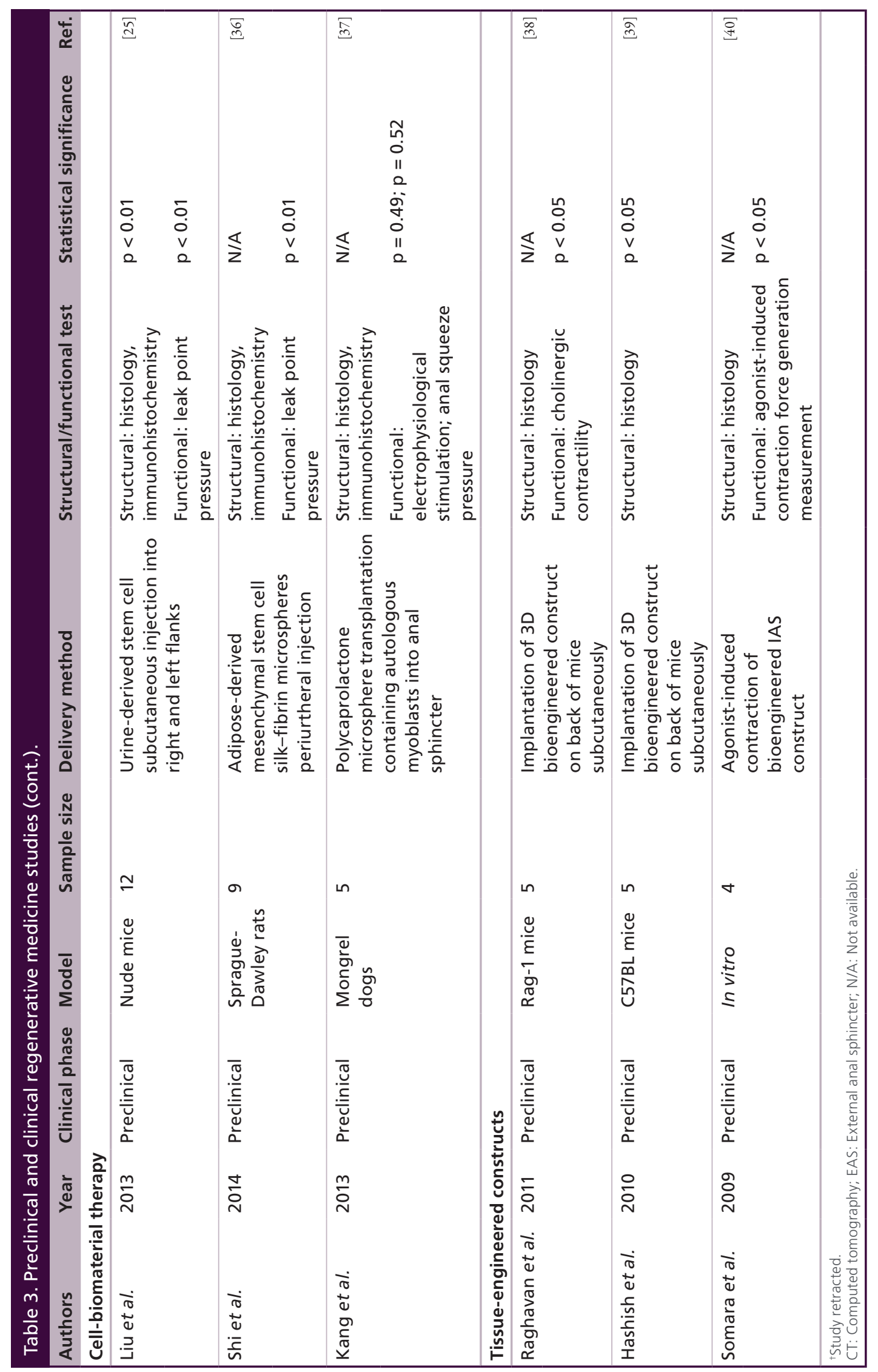


leak point pressure in stress urinary incontinence in a rat model [44]. Cell therapy has also been investigated in preclinical animal models of FI. Lorenzi and colleagues explored the use of bone marrow-derived mesenchymal stem cells to improve repair of sphincter muscle injury in rat models. Cells were delivered using intrasphincteric injections after full thickness internal and external sphincterotomy and end-to-end repair surgery. The results were compared with a control group that underwent end-to-end repair and a saline injection. Results showed new muscle fiber formation with improved contraction in the cell delivery group compared with the control group [35]. While this study appears promising for the use of cell therapy to treat sphincter muscle injury, consideration should be given to the inherent regenerative capacity of the injured tissue in the model used, the ability to precisely target the sphincter muscle of rodents, and whether the effect can be scaled-up for human use. There is little doubt that further preclinical studies such as these are required, not only for improving our understanding of the biological mechanisms involved, but also to help fine tune this approach for future clinical use.

The concept of muscle cell therapy has traditionally been thought to involve stem cell differentiation into functional muscle to regenerate damaged or dysfunctional tissue. However, it is now widely accepted that differentiation alone is insufficient to achieve this aim. It is estimated almost 1 trillion cells are required to replace damaged cardiomyocytes during cardiac failure [45]. When scaling this number of cells into a rat model of cardiac failure, 2 billion cells are required for full restoration - on the assumption that $100 \%$ of the transplanted cells will engraft and differentiate [45]. This figure is much higher than the amount of cells used in the majority of preclinical studies published to date. Another step change in the mind set of research in the field of cell therapy has been recognition of the importance of paracrine trophic factor release from mesenchymal stromal cells in tissue regeneration. The mechanisms behind this involve neutrophil infiltration to site of repair, macrophage activation, satellite cell proliferation and fusion, and neuromuscular junction formation [46]. Further studies are required to understand the paracrine mechanisms underlying tissue regeneration with cell transplantation.

\section{Cell-biomaterial therapy}

Biomaterials have been used in conjunction with cellbased therapies to provide the mechanical and biological properties of host tissue and their extracellular matrix. Biomaterials provide a $3 \mathrm{D}$ scaffold for cells to form new tissue. Ideal properties of biomaterials for soft-tissues such as the sphincter muscle complex include biocompatibility, biodegradation and no significant immune response that may lead to diminished integration and functionality of delivered cells or tissue. The delivery of cell-biomaterial combination therapy has been explored in several animal models and FDA-approved cell therapy clinical trials.

Autologous urine-derived stem cells (UDSCs) have been delivered in a collagen-I hydrogel to the urethral sphincter in mouse model [47]. The UDSCs were genetically modified to express VEGF and implanted with endothelial cells. This study demonstrated the potential to deliver cell-biomaterial combinations together with bioactive factors to regenerate skeletal muscle in a model of urinary incontinence. The study by Shi et al. tested the functional regeneration of the urethral sphincter using adipose-derived mesenchymal stem cells (ADSCs) and silk fibroin microspheres as a bulking agent. ADSCs were found to be superior to the silk fibroin microspheres and showed long-term improvement at 12 weeks in comparison to only a short-term improvement up to 4 weeks seen with the silk fibroin microspheres. The leak point pressure increased to $59.74 \pm 7.88 \mathrm{~cm} \mathrm{H}_{2} \mathrm{O}(\mathrm{p}<0.05)$ with ADSCs, but stayed low at $33.59 \pm 9.52 \mathrm{~cm} \mathrm{H}_{2} \mathrm{O}$ ( $p>0.05$ ) with silk fibroin microspheres. Similarly, the lumen size decreased significantly in ADSCs group, but not with the silk fibroin microsphere group at 12 weeks [36]. This approach may offer clinical value for defects associated with the rhabdosphincter, which measures $0.4-3.7 \mathrm{~mm}$ and is structurally thinner than the EAS, which measures $0.6-1.0 \mathrm{~cm}[4,48]$. The difference in muscle thickness may explain why nonporous scaffolds do not provide sufficient mechanical integrity to support regeneration of larger muscle defects, where neovascularization is needed for nutrient diffusion and removal of waste products to avoid tissue necrosis in the newly formed tissue.

Recently Kang et al. published the first preclinical in vivo study using a bioengineering approach for FI. They injected myoblast cell-loaded polycaprolactone beads into a dog model after inducing anal sphincter injury (partial extraction of approximately $25 \%$ of the posterior IAS/EAS using electrocautery). This study failed to demonstrate any histological or functional improvement postinjection [37].

\section{Tissue-engineered constructs}

In cardiovascular biology, cells have been combined with collagen to construct bioengineered multilayered blood vessels that resemble human arteries and are capable of withstanding physiological pressures [49]. A similar approach has been applied to bioengineering small 3D IAS structures that show possible clinical value as a transplantable tissue [50,51]. Studies have shown the smallscale bioengineered IAS can be engineered using human IAS smooth muscle cells. Functional analysis indicated the IAS rings generate spontaneous basal tone, contrac- 
tion in response to acetylcholine and neovascularization upon implantation onto a subcutaneous pocket on the upper back of mice [38]. While this approach demonstrates feasibility to vascularize the construct, the chosen site of implantation may not be representative of potential vascularization at the site of intended delivery. The use of RAG1 ${ }^{--}$mice with no mature B or T lymphocytes present may also have contributed to no observed immune response to the implanted construct [52]. Suitable sources of donor smooth muscle cells appropriate for bioengineering the construct may prove to be a limitation for this approach if translated to humans in the future. Another limitation is the time required to produce the construct. This would involve isolation and expansion of smooth muscle cells followed by formation of the mature IAS structure. By this stage, the injury may have undergone repair and scarring at the implantation site, which may impede engraftment and contractility of the engineered construct. While a TE approach is very promising, there are significant hurdles to overcome before this can be translated as a viable option for either IAS or EAS regeneration.

\section{Conclusion}

Regenerative medicine holds significant potential to restore muscle function following obstetric injury. Further studies are necessary to verify preclinical findings and to optimize this type of therapeutic approach. An increasing awareness by women of the causes of FI and their willingness to openly discuss this condition, together with the rapid pace of development in the field of regenerative medicine will yield significant improvements in the treatments available for restoring continence.

\section{Future perspective}

The development of regenerative medicine strategies for obstetric trauma-induced FI is fast developing. Preclinical and early-phase clinical research into regeneration of muscle for volumetric muscle loss and urinary incontinence provide great insight to improving targeted, localized cell delivery and the subsequent behavior of implanted cells. There are a number of recently completed and ongoing clinical trials investigating cell therapy that have demonstrated safety, tolerance and feasibility of cell delivery in humans (Table 3). Recently, the focus has shifted towards the use of cells in combination with biomaterials to improve the efficiency and direct delivery of cells to the site of injury in vivo. Although further research is needed to optimize the choice of biomaterial and to understand cell behavior following implantation, the outlook for cellbased therapies for FI appears to be promising for this form of regenerative medicine. Progress of this treatment will undoubtedly be accelerated as other forms of cell therapy become mainstream.

\section{Acknowledgements}

The authors appreciate the technical support of Jaspal Puri and Charles Craddock, UCL Applied Biomedical Engineering Group.

Financial \& competing interests disclosure N Parmar is funded by grants from the UCL Grand Challenge PhD programme and the Sir Halley Stewart Trust. This work was undertaken at UCL/UCLH which receives funding from the Department of Health's NIHR as a Comprehensive Biomedical Research Centre. The authors have no other relevant affiliations or financial involvement with any organization or entity with a financial interest in or financial conflict with the subject matter or materials discussed in the manuscript apart from those disclosed.

No writing assistance was utilized in the production of this manuscript.

\section{Open access}

This work is licensed under the Creative Commons Attribution-NonCommercial 3.0 Unported License. To view a copy of this license, visit http://creativecommons.org/licenses/bync-nd/3.0/

\section{Executive summary}

Pelvic floor maintenance of continence

- Levator ani muscle and anal sphincters help maintain the continence mechanism.

Obstetric trauma-induced fecal incontinence

- Obstetric trauma is a well-recognized cause of fecal incontinence in women.

- Anal sphincter damage remains clinically undetected in $12-35 \%$ of patients.

Regenerative medicine

- Smart biomaterials are being evaluated for their use as 3D scaffolds for tissue regrowth and localized regeneration.

- Autologous cell therapy has been tested in five US FDA-approved clinical trials for muscle regeneration in incontinence with encouraging results.

- Cell-biomaterial therapy is being preclinically evaluated for targeted, localized delivery of cell-attached scaffolds and seems to be the most promising approach.

- Tissue-engineering constructs are being developed for partial or complete replacement of defective muscle. 


\section{References}

Papers of special note have been highlighted as:

- of interest; $\bullet \bullet$ of considerable interest

1 Abrams P, Andersson KE, Birder L et al. Fourth International Consultation on Incontinence Recommendations of the International Scientific Committee: Evaluation and treatment of urinary incontinence, pelvic organ prolapse, and fecal incontinence. Neurourol. Urodyn. 29(1), 213-240 (2010).

2 Rao SS. Pathophysiology of adult fecal incontinence. Gastroenterology 126(1 Suppl. 1), S14-S22 (2004).

3 Rao SS. Diagnosis and management of fecal incontinence. American College of Gastroenterology Practice Parameters Committee. Am. J. Gastroenterol. 99(8), 1585-1604 (2004).

4 Regadas FS, Murad-Regadas SM, Lima DM et al. Anal canal anatomy showed by three-dimensional anorectal ultrasonography. Surg. Endosc. 21(12), 2207-2211 (2007).

5 Frenckner B, Euler CV. Influence of pudendal block on the function of the anal sphincters. Gut 16(6), 482-489 (1975).

6 Bharucha AE, Rao SS. An update on anorectal disorders for gastroenterologists. Gastroenterology 146(1), 37-45 (2014).

7 Niu WX, Qin XY, Lu YQ, Shi NC, Wang CP. Role of intracellular calcium in contraction of internal anal sphincter. World. J. Gastroenterol. 5(2), 183-184 (1999).

8 Herschorn S. Female pelvic floor anatomy: the pelvic floor, supporting structures, and pelvic organs. Rev. Urol. 6(Suppl. 5), S2-S10 (2004).

9 Thekkinkattil DK, Dunham RJ, O'Herlihy S, Finan PJ, Sagar PM, Burke DA. Measurement of anal cushions in idiopathic faecal incontinence. Br. J. Surg. 96(6), 680-684 (2009).

10 Li YD, Xu J H, Lin JJ, Zhu WF. Excisional hemorrhoidal surgery and its effect on anal continence. World J. Gastroenterol. 18(30), 4059-4063 (2012).

11 Khafagy W, El NA, Fouda E et al. Conventional haemorrhoidectomy, stapled haemorrhoidectomy, Doppler guided haemorrhoidectomy artery ligation; post operative pain and anorectal manometric assessment. Hepatogastroenterology 56(93), 1010-1015 (2009).

12 Dyer NH, Dawson AM, Smith B F, Todd IP. Obstruction of bowel due to lesion in the myenteric plexus. $\mathrm{Br}$. Med. J. 1(5645), 686-689 (1969).

13 Fernando RJ, Sultan AH, Radley S, Jones PW, Johanson RB. Management of obstetric anal sphincter injury: a systematic review \& national practice survey. BMC Health Serv. Res. 2(1), 9 (2002).

14 Chaudhary BN, Chadwick M, Roe AM. Selecting patients with fecal incontinence for anal sphincter surgery: the influence of irritable bowel syndrome. Colorectal Dis. 12(8), 750-753 (2010).

15 Eason E, Labrecque M, Marcoux S, Mondor M. Anal incontinence after childbirth. CMAJ 166(3), 326-330 (2002).

16 Borello-France D, Burgio K L, Richter $\mathrm{H}$ et al. Fecal and urinary incontinence in primiparous women. Obstet. Gynecol. 108(4), 863-872 (2006).
17 Sultan AH, Kamm MA, Hudson CN, Thomas JM, Bartram CI. Anal-sphincter disruption during vaginal delivery. N. Engl. J. Med. 329(26), 1905-1911 (1993).

18 Dudding TC, Vaizey CJ, Kamm MA. Obstetric anal sphincter injury: incidence, risk factors, and management. Ann. Surg. 247(2), 224-237 (2008).

19 Starck M, Bohe M, Valentin L. Effect of vaginal delivery on endosonographic anal sphincter morphology. Eur. J. Obstet. Gynecol. Reprod. Biol. 130(2), 193-201 (2007).

20 Hatano K, Inoue H, Kojo T et al. Effect of surface roughness on proliferation and alkaline phosphatase expression of rat calvarial cells cultured on polystyrene. Bone 25(4), 439-445 (1999).

21 Saltzman WM, Olbricht WL. Building drug delivery into tissue engineering. Nat. Rev. Drug Discov. 1(3), 177-186 (2002).

22 Urciuolo A, Quarta M, Morbidoni V et al. Collagen VI regulates satellite cell self-renewal and muscle regeneration. Nat. Commun. 4, 1964 (2013).

23 Madaghiele M, Sannino A, Yannas IV, Spector M. Collagen-based matrices with axially oriented pores. J. Biomed. Mater. Res. A 85(3), 757-767 (2008).

24 Oliveira SM, Ringshia RA, Legeros RZ et al. An improved collagen scaffold for skeletal regeneration. J. Biomed. Mater. Res. A 94(2), 371-379 (2010).

25 Liu G, Wang X, Sun X, Deng C, Atala A, Zhang Y. The effect of urine-derived stem cells expressing VEGF loaded in collagen hydrogels on myogenesis and innervation following after subcutaneous implantation in nude mice. Biomaterials 34(34), 8617-8629 (2013).

26 Beier J P, Klumpp D, Rudisile M et al. Collagen matrices from sponge to nano: new perspectives for tissue engineering of skeletal muscle. BMC Biotechnol. 9, 34 (2009).

27 Mosesson MW. Fibrinogen and fibrin structure and functions. J. Thromb. Haemost. 3(8), 1894-1904 (2005).

28 Arkudas A, Pryymachuk G, Hoereth T et al. Dose-finding study of fibrin gel-immobilized vascular endothelial growth factor 165 and basic fibroblast growth factor in the arteriovenous loop rat model. Tissue Eng. Part A 15(9), 2501-2511 (2009).

29 Zhao C, Yao J, Masuda H, Kishore R, Asakura T. Structural characterization and artificial fiber formation of Bombyx mori silk fibroin in hexafluoro-iso-propanol solvent system. Biopolymers 69 (2), 253-259 (2003).

30 Wang Y, Rudym DD, Walsh A et al. In vivo degradation of three-dimensional silk fibroin scaffolds. Biomaterials 29(24-25), 3415-3428 (2008).

31 Henderson J, Ferguson MW, Terenghi G. The reinnervation pattern of wounds and scars after treatment with transforming growth factor beta isoforms. J. Plast. Reconstr. Aesthet. Surg. 65(4), e80-e86 (2012).

32 Yiou R, Yoo JJ, Atala A. Restoration of functional motor units in a rat model of sphincter injury by muscle precursor cell autografts. Transplantation 76(7), 1053-1060 (2003).

33 StrasserH, Marksteiner R, Margreiter E et al. Autologous myoblasts and fibroblasts versus collagen for treatment 
ofstress urinary incontinence in women: a randomised controlled trial. Lancet 369(9580), 2179-2186 (2007).

34 Frudinger A, Kolle D, Schwaiger W, Pfeifer J, Paede J, Halligan S. Muscle-derived cell injection to treat anal incontinence due to obstetric trauma: pilot study with 1 year follow-up. Gut 59(1), 55-61 (2010).

-• The only autologous cell therapy clinical study for obstetric trauma-induced fecal incontinence.

35 Lorenzi B, Pessina F, Lorenzoni P et al. Treatment of experimental injury of anal sphincters with primary surgical repair and injection of bone marrow-derived mesenchymal stem cells. Dis. Colon. Rectum 51(4), 411-420 (2008).

36 Shi L B, Cai H X, Chen L K et al. Tissue engineered bulking agent with adipose-derived stem cells and silk fibroin microspheres for the treatment of intrinsic urethral sphincter deficiency. Biomaterials 35(5), 1519-1530 (2014).

37 Kang SB, Lee H S, Lim J Y et al. Injection of porous polycaprolactone beads containing autologous myoblasts in a dog model of fecal incontinence. J. Korean Surg. Soc. 84(4), 216-224 (2013).

- The only cell-biomaterial therapy study for external anal sphincter regeneration.

38 Raghavan S, Gilmont R R, Miyasaka E A et al. Successful implantation of bioengineered, intrinsically innervated, human internal anal sphincter. Gastroenterology 141(1), 310-319 (2011).

39 HashishM, Raghavan S, Somara S et al. Implantationof a bioengineered internal anal sphincter. J. Pediatr. Surg. 45(1), 52-58 (2010).

40 SomaraS, Gilmont RR, Dennis RG, Bitar KN. Bioengineered internal anal sphincterderived from isolated human internal anal sphincter smooth muscle cells. Gastroenterology 137(1), 53-61 (2009).

41 Itescu S, Schuster MD, Kocher AA. New directions in strategies using cell therapy for heart disease. J. Mol. Med. 81(5), 288-296 (2003).

42 Smythe GM, Hodgetts SI, Grounds MD. Immunobiology and the future of myoblast transfer therapy. Mol. Ther. 1(4), 304-313 (2000).
43 Smythe GM, Hodgetts SI, Grounds MD. Problems and solutions in myoblast transfer therapy. J. Cell. Mol. Med. 5(1), 33-47 (2001).

- Demonstrates the immunological disadvantages of 2D cultured cells for therapy.

44 Fu Q, Song XF, Liao GL, Deng CL, Cui L. Myoblasts differentiated from adipose-derived stem cells to treat stress urinary incontinence. Urology 75(3), 718-723 (2010).

45 Mazo M, Gavira JJ, Pelacho B, Prosper F. Adipose-derived stem cells for myocardial infarction. J. Cardiovasc. Transl. Res. 4(2), 145-153 (2011).

46 Ciciliot S, Schiaffino S. Regeneration of mammalian skeletal muscle. Basic mechanisms and clinical implications. Curr. Pharm. Des. 16(8), 906-914 (2010).

47 Liu G, Wang X, Sun X, Deng C, Atala A, Zhang Y. The effect of urine-derived stem cells expressing VEGF loaded in collagen hydrogels on myogenesis and innervation following after subcutaneous implantation in nude mice. Biomaterials 34(34), 8617-8629 (2013).

48 Klauser A, Frauscher F, Strasser $\mathrm{H}$ et al. Age-related rhabdosphincter function in female urinary stress incontinence: assessment of intraurethral sonography. J. Ultrasound Med. 23(5), 631-637 (2004).

49 Weinberg C B, Bell E. A blood vessel model constructed from collagen and cultured vascular cells. Science 231(4736), 397-400 (1986).

50 Hecker L, Baar K, Dennis R G, Bitar K N. Development of a three-dimensional physiological model of the internal anal sphincter bioengineered in vitro from isolated smooth muscle cells. Am. J. Physiol. Gastrointest. Liver Physiol. 289(2), G188-G196 (2005).

-• The first demonstration of bioengineered tissue for replacement of the internal anal sphincter.

51 Bitar K N, Raghavan S. Intestinal tissue engineering: current concepts and future vision of regenerative medicine in the gut. Neurogastroenterol. Motil. 24(1), 7-19 (2012).

52 Mombaerts P, Iacomini J, Johnson R S, Herrup K, Tonegawa S, Papaioannou V E. RAG-1-deficient mice have no mature B and T lymphocytes. Cell 68(5), 869-877 (1992). 